\title{
Inactivation Technology for Pitch Doubling Lithography
}

\author{
Jun Hatakeyama, Masaki Ohashi, Youichi Ohsawa, \\ Kazuhiro Katayama and Yoshio Kawai \\ New Functional Materials Research Center, Shin-Etsu Chemical Co., Ltd. \\ 28-1 Nishifukushima, Kibiki, Nakakubiki, Niigata 942-8601, Japan \\ jun.hata@.shinetsu.jp
}

\begin{abstract}
We propose novel inactivation technologies which improve resolution. Base generators have been developed, which inactivate acid by thermal treatment or exposure. This thermal inactivation technology realizes simple litho-inactivation-litho-etch (LILE) process with good fidelity. After $1^{\text {st }}$ patterning, acid is inactivated by amine released from the thermal base generator under low temperature baking of less than $150^{\circ} \mathrm{C}$. Just adding one simple low temperature bake process, LILE has two advantages; i) keeping high throughput, and ii) avoidance of pattern deformation. $32 \mathrm{~nm}$ line and space $(1 \& \mathrm{~s})$ pattern is successfully delineated by pitch split double patterning. The inactivation technology has been expanded to frequency doubling. Photo base generator (PBG) is used to inactivate acid generated by exposure. Acid concentration in both of low and high exposed area is precisely controlled by base generation efficiency of PBG. The dual tone resist, which has positive tone dissolution property at low dose region and negative tone at high dose, splits a line in two and successfully delineates $32.5 \mathrm{~nm} 1 \&$ s pattern using $65 \mathrm{~nm} 1 \& \mathrm{~s}$ mask with single exposure.
\end{abstract}

Keywords: Inactivation, Base generator, Double patterning, LILE, Dual tone resist, Frequency doubling

\section{Introduction}

In order to push forward the finer IC manufacturing, both the minimum critical dimension and the pattern pitch of the circuit feature must be reduced. Immersion lithography contributed to accelerate $45 \mathrm{~nm}$ node device production. The coming of extreme ultraviolet (EUV) lithography is desired to realize beyond $22 \mathrm{~nm}$ node, however it has many technical issues to overcome. Post $40 \mathrm{~nm}$ technology is facing thresholds beyond which conventional photolithography process cannot transcend.

Double patterning technology is one of the candidates to break the wavelength brick wall ${ }^{1)}$. Over the past few years a considerable number of studies have been carried out to apply double patterning. Self-aligned double patterning (SADP) of sidewall spacer is approaching manufacturing stage of flash memory devices. Litho etch litho etch (LELE) double patterning has been investigated for DRAM and logic devices. Litho litho etch (LLE) including additional freezing step have been proposed such as coating materials cure, $\mathrm{HBr} / \mathrm{Ar}$ plasma cure, ion implantation cure and vacuum ultra violet (VUV) irradiation cure. ${ }^{2), 3), 4), 5)}$ Simpler LLE processes have been developed employing thermal freezing chemistry in $1^{\text {st }}$ resist $^{(6)}$ or freezing free Posi/Posi process with alcohol solvent of low PEB temperature $2^{\text {nd }}$ resist ${ }^{7}$.

Cost of ownership is a major concern for double patterning. Therefore, process simplicity is 
desired for increasing productivity and decreasing process cost. Double exposure concept have been brought by non-linear resist and contrast enhancement layer (CEL). ${ }^{8)}$, 9) The simplest process is a frequency doubling in the conventional process of single exposure and single development; positive-negative hybrid dual tone resist has been proposed. ${ }^{10)}$

In this paper, we report rational freezing free materials for LLE application. We also report novel dual tone resist, which realizes frequency doubling by single exposure and single development. The concept, mechanism and optimization of dual tone resist are discussed on simulation and experimental patterning results.

\section{Method}

\subsection{Materials}

Polymethacrylate derivative, which has acid labile and lactone adhesion units, was used for $1^{\text {st }}$ resists (ㅌelf hypnosis $\underline{\operatorname{ArF}}$ resist for double patterning: SHAD). Base polymer, photo acid generator (PAG), quencher and thermal base generator (TBG) were mixed in propylene glycol 1-monomethyl ether 2-acetate (PGMEA) / cyclohexanone solvent. Carbamate derivatives were used for TBG. Polymethacrylate derivative, including acid labile and hexafluoroalcohol adhesion units, was used for 2 nd patterning resists (Joist for $\underline{\text { ArF }}$ double exposure: JADE). Base polymer, PAG and quencher were mixed in alcohol solvent.

Double Frequency ArF resist: DFAR was formulated with PAG, quencher, PBG, polymethacrylate derivative and PGMEA/ cyclohexanone solvent. Triphenylsulfonium salt derivative was used for PAG.

\subsection{Lithography process}

A CLEAN TRACK ${ }^{\mathrm{TM}}$ LITHIUS $^{\mathrm{TM}}$ i+ (Tokyo Electron Ltd.) was used for SHAD, JADE DFAR apply, bake and development. A Nikon immersion scanner (NSR-S610C) was used for pattern formation of SHAD, JADE and DFAR.

SHAD, JADE and DFAR development was carried out in $2.38 \mathrm{wt} \%$ tetramethylammonium hydroxide (TMAH) aqueous solution as developer with single puddle for $30 \mathrm{sec}$.

\subsection{Measurement}

Film thickness was measured with Lambda ACE VM-3010 (Dainippon Screen Manufacture Co. Ltd.). Photo resist line width was measured with a CG-4000 (Hitachi hi-technologies Co.) and cross section pattern was observed with a S-4300 (Hitachi hi-technologies Co.).

Photo acid and base generation were measured on quarts wafers on absorbance at $522 \mathrm{~nm}$ of coumarin 6, with ArF exposure tool of an ArFES (Litho Tech Japan Ltd.).

\subsection{Simulation}

Optical simulation was carried out on PROLITH $^{\mathrm{TM}}$ (KLA-Tencor Co.) and resist profile simulation was run on Shin-Etsu in house simulator ${ }^{11)}$.

\section{Results and Discussion}

\subsection{LILE Process Flow}

A positive resist (SHAD) containing thermal base generator (TBG) for $1^{\text {st }}$ patterning and an alcohol solvent positive resist (JADE) for $2^{\text {nd }}$ patterning are employed (Figure: 1). After $1^{\text {st }}$ patterning, inactivation bake treatment is carried out by acid neutralization with amine from the thermal base generator.

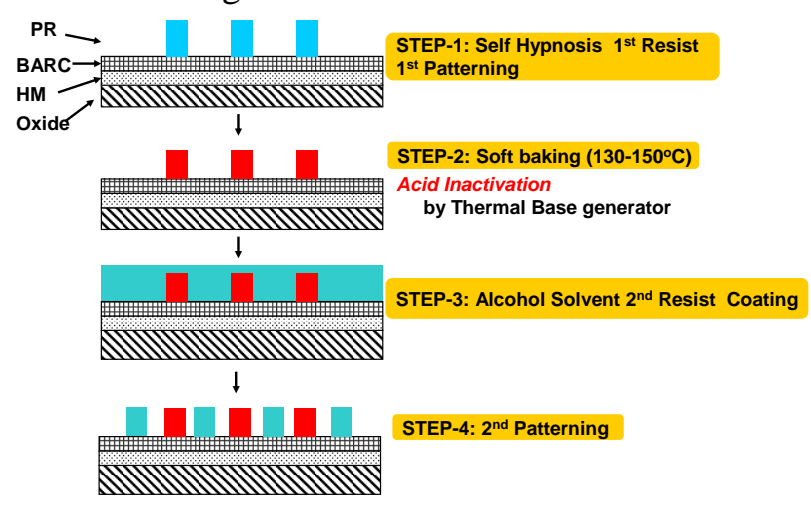

Figure1: LILE Process flow

\subsection{Materials and process}

3.2.1 Pattern Retention Ability of Self Hypnosis Resist (SHAD)

A 70nm 1\&s SHAD pattern was formed on BARC (Figure 2a). Figure $2 b$ shows the SHAD pattern after the steps of inactivation bake $\left(130^{\circ} \mathrm{C} / 60 \mathrm{~s}\right)$, JADE coat, flood exposure $\left(30 \mathrm{~mJ} / \mathrm{cm}^{2}\right)$, PEB and development. JADE dissolved and SHAD pattern appeared after development. Skipping inactivation bake, the 
SHAD pattern disappeared (Figure 2c). The result indicates that the addition of TBG and the inactivation bake treatment is effective to keep 1st pattern shape.

a)

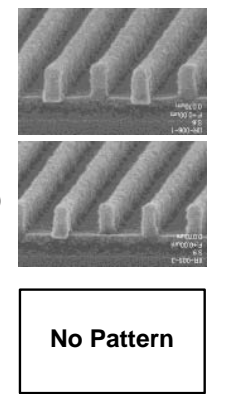

1st Pattern

Inactivation Bake of 1 st Pattern $\left(130^{\circ} \mathrm{C} / 60 \mathrm{~s}\right)$

$\Rightarrow 2^{\text {nd }}$ Resist Coat $\Rightarrow$ Flood Exposure $\left(30 \mathrm{~mJ} / \mathrm{cm}^{2}\right)$ $\Rightarrow \mathrm{PEB} \Rightarrow$ Development

$\Rightarrow \quad 2^{\text {nd }}$ Resist Coat $\Rightarrow$ Flood Exposure $\left(30 \mathrm{~mJ} / \mathrm{cm}^{2}\right)$ $\Rightarrow$ PEB $\Rightarrow$ Development Skip Inactivation Step

Figure 2: $1^{\text {st }}$ pattern retention ability after $2^{\text {nd }}$ development

3.2.2 Hole fabrication by cross line of double patterning

A delineation of hole pattern by double patterning of $\mathrm{X}-\mathrm{Y}$ cross line has been proposed ${ }^{12)}$. The combination of dipole illumination and polarization creates very high optical contrast, it is promising technology to fabricate $0.3 \mathrm{k}_{1}$ hole.

$1^{\text {st }}$ SHAD was printed in vertical line on a $100 \mathrm{~nm}$ film thickness and $2^{\text {nd }} \mathrm{JADE}$ was cross over horizontal line on a $65 \mathrm{~nm}$ film thickness. Illumination condition was NA1.30, dipole, polarization, $6 \%$ half-tone phase shifting mask (HT-PSM). Figure 3 shows 40nm hole pattern with constant exposure dose on $1^{\text {st }}$ SHAD and variation dose on $2^{\text {nd }} \mathrm{JADE}$. No $\mathrm{CD}$ variation in $1^{\text {st }}$ pattern was observed with variation of $2^{\text {nd }}$ exposure dose. This result indicates that acid on $2^{\text {nd }}$ exposure is efficiently inactivated by TBG.

Figure 4 shows $40 \mathrm{~nm}$ hole with constant exposure dose and different focus position. A square hole pattern was formed and more than $0.2 \mu \mathrm{m}$ depth of focus (DOF) margin was obtained.

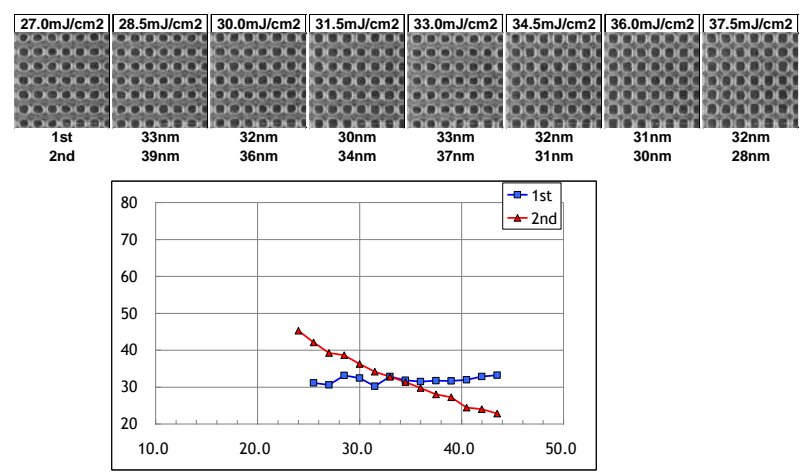

Figure 3: $40 \mathrm{~nm}$ hole with variety of $2^{\text {nd }}$ exposure dose

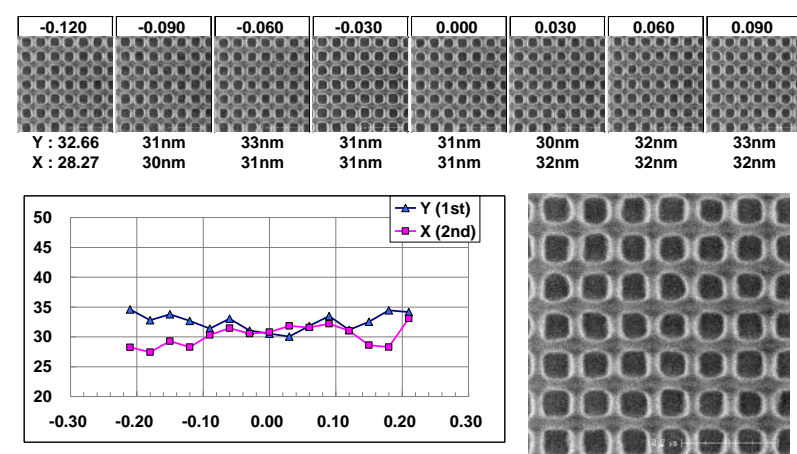

Figure 4: 40nm hole with variety of focus position

\subsubsection{Pitch Split by Double patterning}

Pitch split by double patterning is attractive approach to breaking trough the diffraction resolution limit at $\mathrm{k}_{1}$ value of 0.25 .

$128 \mathrm{~nm}$ pitch of $1^{\text {st }}$ SHAD pattern was printed on $70 \mathrm{~nm}$ thickness and $2^{\text {nd }} \mathrm{JADE}$ was printed with $32 \mathrm{~nm}$ shift position on $65 \mathrm{~nm}$ thickness. Illumination condition is NA1.20, polarization, $6 \%$ HT-PSM. $32 \mathrm{~nm} 1 \& \mathrm{~s}$ pattern was delineated by pitch split double patterning (Figure 5).
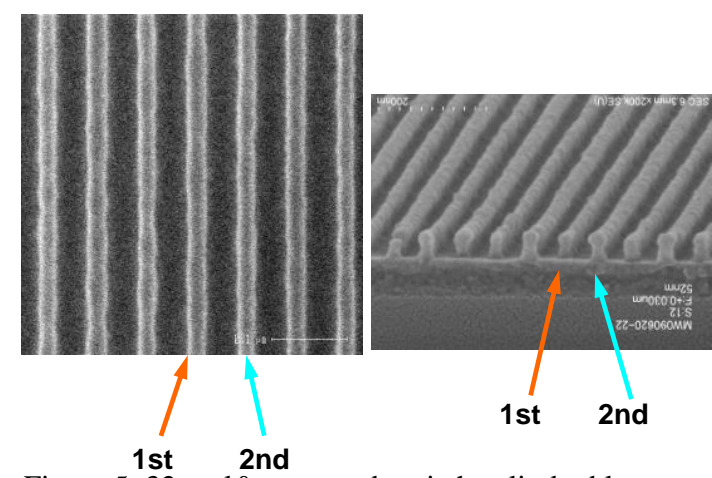

Figure 5: $32 \mathrm{~nm} 1 \&$ s pattern by pitch split double patterning

\subsubsection{Dual Tone Concept by Photo Inactivation}

Dual tone resist has two thresholds and split a line in two. It has possibility of frequency doubling by single exposure (Figure 6). Dual tone resist is the most attractive approach to realizing pitch split without additional freezing process and exposure.

Steven J. Holmes et al. showed dual tone resist by hybrid of positive-tone and negative-tone. ${ }^{10)}$ The hybrid of positive and negative had been formulated partially acid labile protected polyhydroxystyrene, PAG and cross linker. However, in case of polymethacrylate based $\mathrm{ArF}$ resist, it is inconsistent with reality in negative tone, because of low reactivity in cross-link reaction of carboxylic acid. 
a

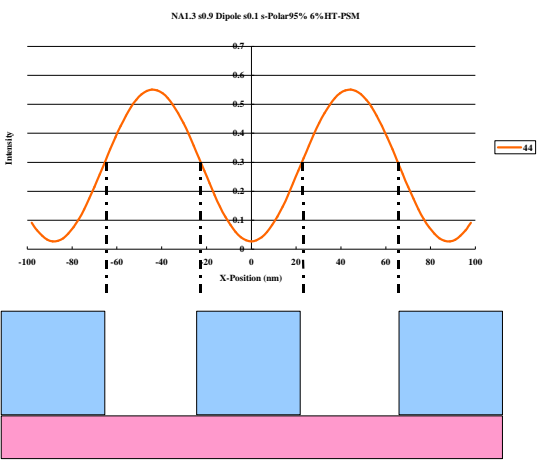

b

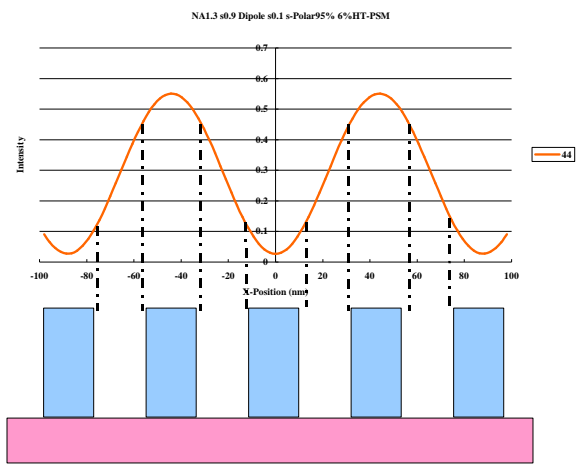

Figure 6: Aerial image and photo resist profile;

conventional positive resist (a) and dual tone resist (b)

The photo base generators have been developed $^{13,14), 15)}$ and their applications ${ }^{16,17)}$ were reported for negative tone and image reversal. For tone inversion, the idea of acid inactivation by base generators is reasonable approach.

Figure 7 (a) shows calculation result of acid, base and active acid concentration with different base generation efficiency. Acid or base from PAG or PBG is calculated in eq.1. The $\phi$ is expressed by Dill's $C$ parameter. The active acid is calculated in eq. 2 .

In the case that the following two conditions are satisfied, 1) PBG efficiency is lower than that of PAG, and 2) a total molar amount of PBG and additive quencher is $10 \sim 80 \%$ higher than PAG, both of an active acid increased region and an active acid decreased region versus dose are appeared. These regions with increase and decrease of the active acid provide positive-tone and negative tone patterns, respectively.

Simulations of dissolution rate curves are shown in Figure 7 (b). The simulation model is based on parallel reaction of acid-base and acid catalyst deprotection $^{11)}$. A set of PAG $(\phi=0.05)$ and PBG $(\phi=0.01)$ shows steeper active acid slope in convex curve (a-2), slower dissolution rate and higher gamma in negative-tone (b-2) than a set of PAG and PBG of small efficiency difference (b-1) and large efficiency difference (b-3). Moderate gap between acid and base generation efficiency is necessary to improve negative contrast. The dissolution contrast of negative-tone is lower than that of positive-tone, which is expressed in higher dissolution rate and slow gamma in negative-tone even in the optimum PAG and PBG efficiency.

Acid or Base generation $=1-\exp (-\phi *$ Dose $)$ ...eq. 1

$\phi$; acid and base generation efficiency

Active acid = Acid from PAG - Base from PBG Additive quencher

\subsubsection{Effect of Positive-Negative Dose Gap on Resists Pattern Profile Simulation}

Positive and negative exposure dose can be variable by changing amount of additive quencher or PBG. Positive and negative exposure dose gap becomes narrow with an increase of the quencher amount or PBG. Figure 8 shows relationship of positive and negative dose gap and resists profiles. In Figure 8, optimum PAG $(\phi=0.05)$ and PBG $(\phi=0.01)$ efficiently, which is result from Figure 7 , and different quencher amount are used for simulation parameter. The resists profiles were calculated on NA1.35, dipole Illumination, $44 \mathrm{~nm}$ $1 \&$ s $6 \%$ HT-PSM. Z-axis in right pictures is reciprocal number of dissolution rate; it shows resist pattern profile. In the case that negative/positive dose ratio is 8 , negative pattern do not appear. That dose ratio of 3 gives wide negative pattern. Optimum dose ratio is 5 . This result suggests that it has a possibility to print $22 \mathrm{~nm} 1 \& \mathrm{~s}$ pattern from $44 \mathrm{~nm}$ mask. The line width of negative seems wider and dissolution rate is a little higher than positive. The profile difference between positive and negative is caused by low contrast of negative-tone. The simulation was carried out on the parameters, in which neutralization rate constants of acid-quencher and acid-generated base are the same. If the rate constant of acid-generated base is changed to 
(a)
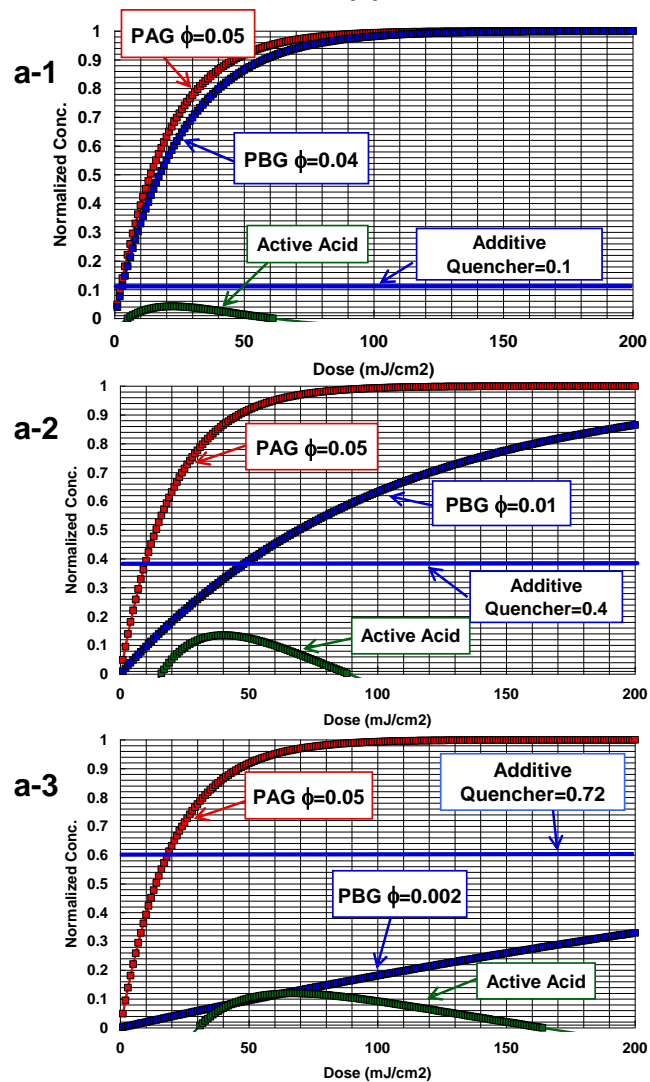

(D)
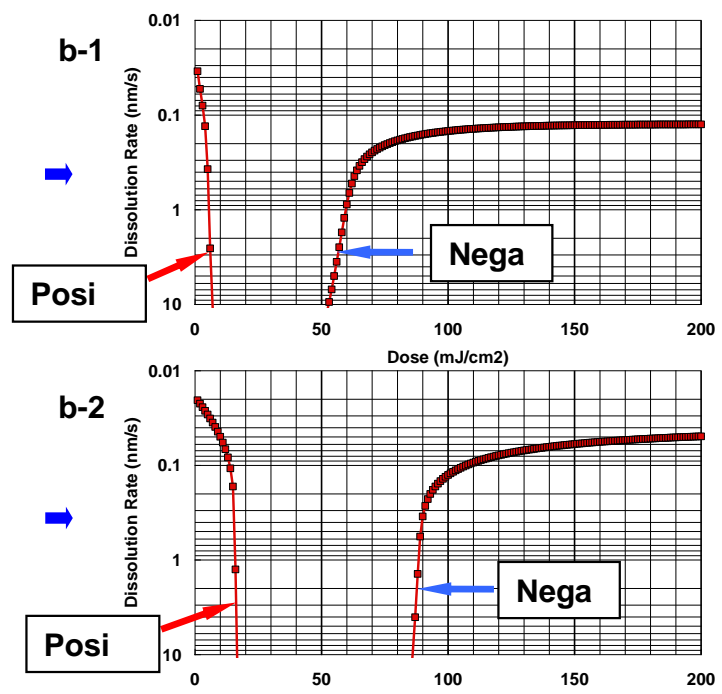

Dose $(\mathrm{mJ} / \mathrm{cm} 2)$

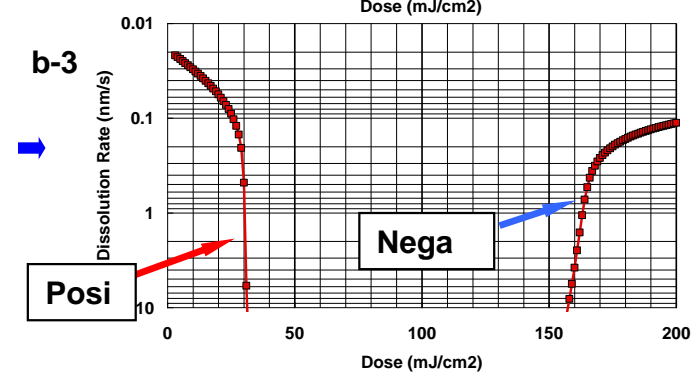

Figure 7: Acid, base and active acid concentration calculation result with different base generation efficiency (a) and dissolution contrast curves (b)
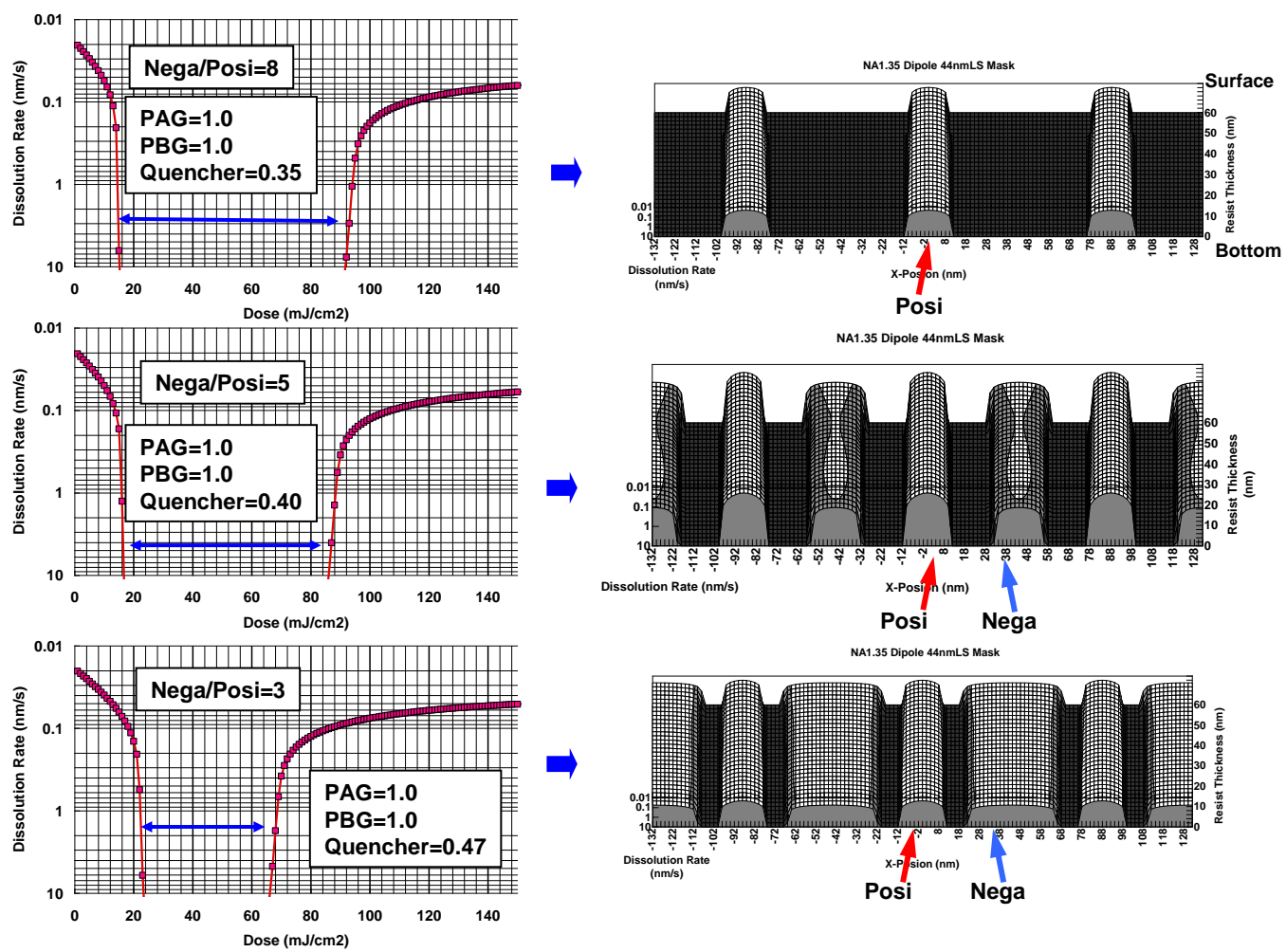

Figure 8: Simulation of contrast curves with different positive-negative exposure dose gap (a) and resist profiles (b) 


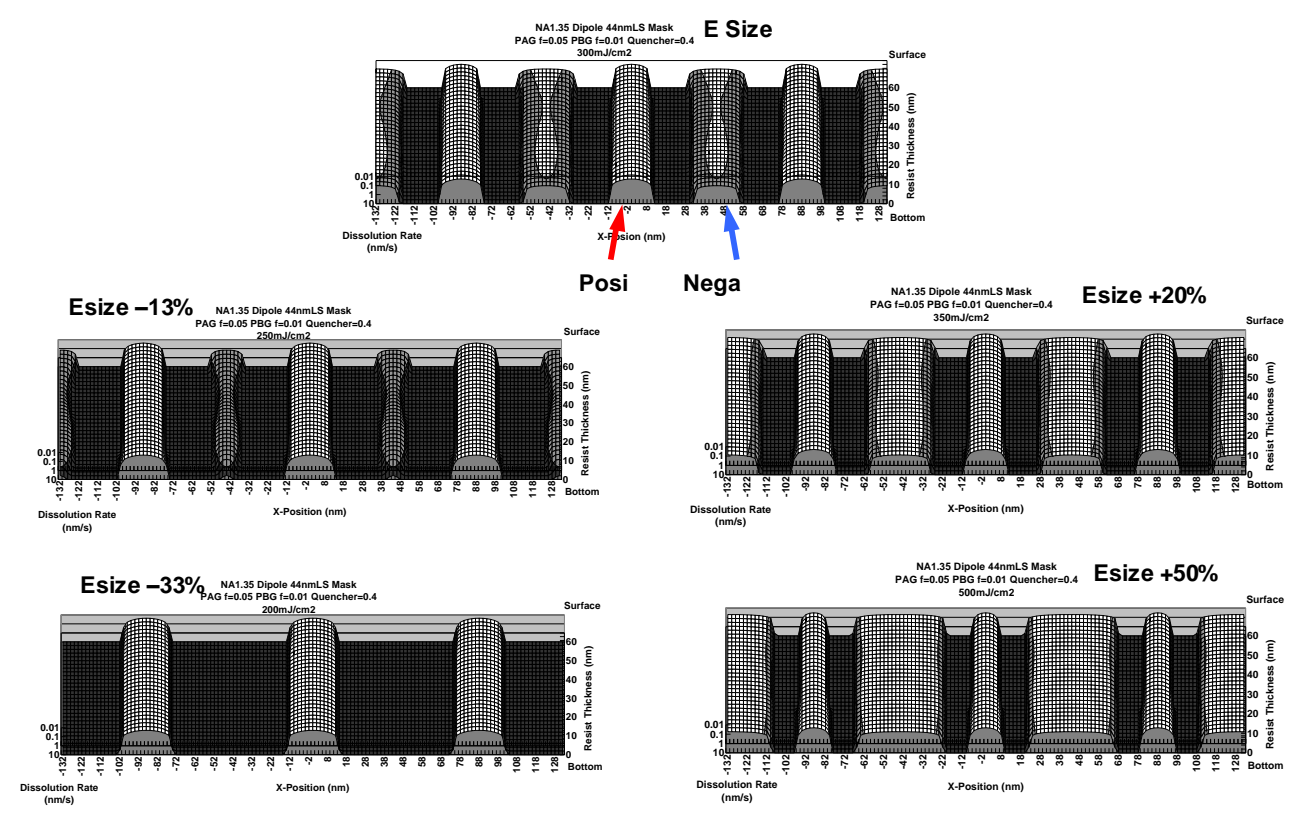

Figure 9: Simulation of frequency doubling resist profiles with variety exposure dose

higher, the negative contrast would be increased.

A contrast enhancement of negative-tone in resist materials development is necessary to harmonize positive and negative profiles.

\subsubsection{Dose Variation on Resist Profile Simulation}

Resist profile simulation with variety of exposure dose is shown in Figure 9. The resists profiles were calculated on NA1.35, dipole Illumination, $44 \mathrm{~nm} \quad 1 \& \mathrm{~s} \quad 6 \%$ HT-PSM. Positive-tone line becomes slim with an increased exposure dose, while negative-tone line becomes fat. Exposure latitude of negative is narrow, compared to positive.

\subsubsection{Acid and Base Generation Efficiency Measurement}

Coumarin- 6 is an acid sensitive dye that exhibits a change in the visible absorption when protonated. The intensity of the peak at $\sim 522 \mathrm{~nm}$ corresponds to the amount of protonated coumarin- 6 and is therefore a measure of the amount of acid in the film.

PAG, coumarine 6 and polymethacrylate were mixed in PGMEA/PGME/ $\gamma$-Butyrolactone mixture solvent. For base detection, camphorsulfonic acid, PBG, coumarine 6 and polymethacrylate are mixed in the solvent above.
PAG or PBG containing solution were spun coat on quartz substrate and baked at $100^{\circ} \mathrm{C} / 60 \mathrm{~s}$. After ArF exposure, $522 \mathrm{~nm}$ absorbance was measured with a spectrophtometer. Normalized acid or base concentrations were calculated by the $522 \mathrm{~nm}$ absorbance. The measurement results, fitting curves and C-parameters $f$ of acid generation from triphenylsulfonium salt and base generation from PBG-1 are illustrated in Figure 10. The base generation efficiency is one fifth of the acid generation, and this value is better for negative-tone contrast, as mentioned above.

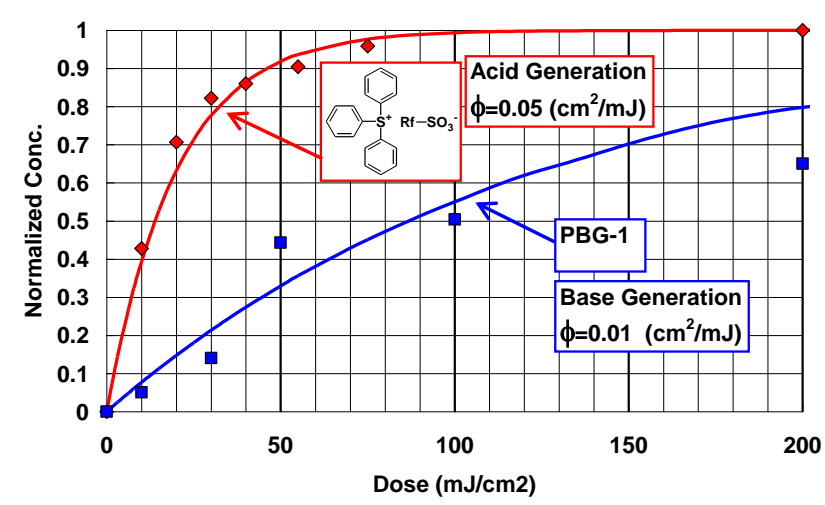

Figure 10: Measurement result of acid and base generation efficiency 
3.2.8 Optimization of Positive-Negative Dose Gap on Patterning Property

Patterning pictures and contrast curves with different positive-negative dose gap are shown in Figure 11. The positive-negative dose gap was changed by different quencher amount.

$60 \mathrm{~nm}$ thickness of DFAR was spun coat on BARC. Illumination condition was NA1.05, dipole, polarization and 6\% HT-PSM.

The gamma of negative was slower and the film loss of negative was bigger than that of positive. In case of negative dose divided by positive dose equals 5; it gave frequency doubling of $50 \mathrm{~nm}$ line from $100 \mathrm{~nm}$ mask. The simulation result was proved by experimental patterning.

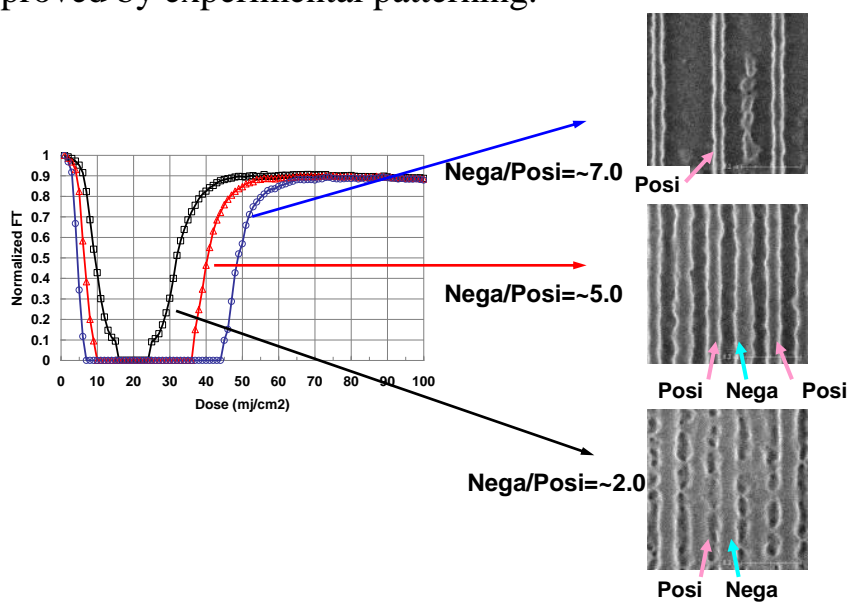

Figure11: Contrast curves and patterning result of dual tone resist

\subsubsection{Patterning Result of Frequency Doubling} Resist

Figure 12 shows patterning result of dual tone resist with 100,80 and $65 \mathrm{~nm}$ mask. $60 \mathrm{~nm}$ thickness of DFAR was spun coat on multi layer of spin on glass and spin on carbon. Illumination condition was NA1.05, dipole, polarization and 6\% HT-PSM.

Negative lines appeared at positive space and increased width wider with an increase exposure dose. $50 \mathrm{~nm}$ and $40 \mathrm{~nm}$ line patterns were printed by single exposure with $100 \mathrm{~nm}$ and $80 \mathrm{~nm}$ mask and partially printed at $32.5 \mathrm{~nm}$ line pattern with $65 \mathrm{~nm}$ mask. There is a possibility for resolving $32 \mathrm{~nm} 1 \&$ s and finer by material improvement.

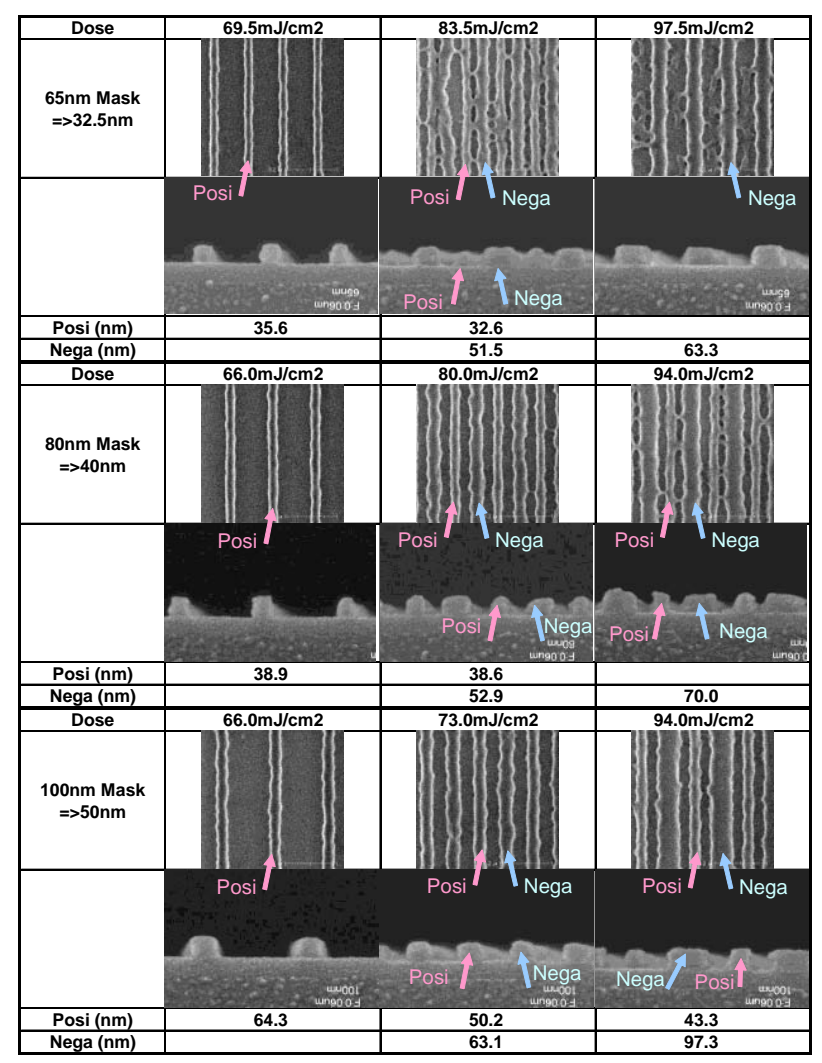

Figure12: SEM pictures of frequency doubling resist at various exposure dose and mask size

\section{Acknowledgements}

The authors would like to acknowledge Mr. T. Ishihara for his advice and encouragement. We also wish to thank the resist development team of the New Functional Materials Research Center for the technical support

\section{References}

1. M. Maenhoudt, J. Versluijs, H. Struyf, J. Van Olmen, and M. Van Hove, Proc. SPIE 5754, (2005) p1508

2. Masafumi Hori, Tomoki Nagai, Atsushi Nakamura, Takayoshi Abe, Gouji Wakamatsu, Tomohiro Kakizawa, Yuusuke Anno, Makoto Sugiura, Shiro Kusumoto, Yoshikazu Yamaguchi and Tsutomu Shimokawa, Proc. SPIE 6923, (2008) p69230H-1

3. Arnaud Bazin, Erwine Pargon, Xavier Mellahoui, Damien Perret, Bénédicte Mortini and Olivier Joubert, Proc. SPIE 6923, (2008) p692337-1 
4. Hiroko Nakamura, Takeshi Shibata, Katsumi Rikimaru, Sanae Ito, Satoshi Tanaka and Soichi Inoue, Proc. SPIE 6923, (2008) p692322-1

5. Nikolaos Bekiaris, Hiram Cervera, Junyan Dai, Ryoung-han Kim, Alden Acheta, Thomas Wallow, Jongwook Kye, Harry J. Levinson, Thomas Nowak and James Yu, Proc. SPIE 6923, (2008) p692321-1 6. Kuang-Jung Rex Chen, Wu-Song Huang, Wai-Kin Li, and P. Rao Varanasi, Proc. SPIE 6923, (2008) p69230G-1

7. Tsuyoshi Nakamura, Masaru Takeshita, Satoshi Maemori, Ryusuke Uchida, Ryouichi Takasu and Katsumi Ohmori, Proc. SPIE 7273, (2009) p72304-1

8. Hiroshi Ooki, Masaya Komatsu and Masato Shibuya, Jpn. J. Appl. Phys. Vol.33 (1994) L177

9. Ryoung-han Kim and Harry Levinson, J. Vac. Sci. Technol. B25 (6), Nov/Dec (2007) p2466

10. Steven J. Holmes, Toshiharu Furusawa, Mark

C. Hakey, David V. Horak and Paul A. Rabidoux,
Proc. SPIE 3678, (1999) p348

11. Jun Hatakeyama, Shigehiro Nagura and Toshinobu Ishihara, J. Photopolym. Sci. and Technol. Vol.13, No.4 (2000) p519

12. Hiroko Nakamura, Yasunobu Onishi, Kazuya Sato, Satoshi Tanaka, Shoji Mimotogi, Koji Hashimoto and Soichi Inoue, Proc. SPIE 5377, (2004) p255

13. James F. Cameron and Jean M. Frechet, J. Am. Chem. Soc., 113, (1991) p4303

14. James F. Cameron, C. Grant Willson and Jean M. Frechet, J. Chem. Soc., Perkin Trans. 1, (1997) p 2429

15. Masahiro Tsunooka, Kanji Suyama, Haruyuki Okamura and Masamitsu Shirai, J. Photopolym. Sci. and Technol., Vol.19, No.1, (2006) p65

16. K. A. Graziano, S.D. Thompson and M.R. Winkle, Proc. SPIE 1466, (1991) p75

17. E. Richter, K. Elian, S. Hien, E. Kuhn, M. Sebald and M. Shirai, Proc. SPIE 3999, (2000) p91 